Pol. Con. (Edición núm. 34) Vol. 4, No 6, junio 2019.

Polo del Conocimiento es una publicación Multidisciplinaria, que difunde trabajos científicos originales, ensayos y revisiones bibliográficas vinculadas con las Ciencias de la Salud, Ciencias Sociales y Políticas, Ciencias de la Educación, Ciencias de la Comunicación, Ciencias Técnicas y Aplicadas, Ciencias Naturales, Artes y Letras, Ciencias Económicas y Empresariales, Ciencias Matemáticas, Ciencias de la Computación y Ciencias del Deporte. Esta publicación está dirigida a investigadores, científicos, estudiantes de pregrado, postgrado y postdoctorado, docentes y profesionales que deseen visualizar sus procesos investigativos $\mathrm{y}$ contribuir al crecimiento del saber académico y científico.

Revista científico-profesional en formato digital, perteneciente a la Casa Editora del Polo, de Manta, Ecuador. Posee una frecuencia de salida mensual e incluye la posibilidad de publicar números monográficos y especiales.

La Revista posee un diseño que asegura la eficiencia y rapidez en el proceso de evaluación de los trabajos, teniendo como premisa esencial el rigor científico, técnico y profesional. Todos los textos enviados son sometidos a una revisión por pares en la modalidad de doble ciego. Es una revista de acceso abierto (http://polodelconocimiento.com/ojs/index. php/es).

\section{EQUIPO EDITORIAL}

\section{Director}

Dr. Víctor R. Jama-Zambrano, Universidad Laica Eloy Alfaro de Manabí, Extensión Chone, Chone, Ecuador, Casa Editora del Polo (CASEDELPO), Manta, Ecuador

\section{Editor}

Dr. Josía Jeseff Isea-Argüelles, Universidad Nacional Experimental Francisco de Miranda, Fundación Koinonía, Instituto de Investigación y Estudios Avanzados koinonía. Santa Ana de Coro, Venezuela.

Dr. Julio Juvenal Aldana-Zavala, Universidad Nacional Experimental Francisco de Miranda, Fundación Koinonía, Instituto de Investigación y Estudios Avanzados koinonía. Venezuela.

Néstor Darío Suarez-Montes, Casa Editora del Polo (CASEDELPO), Manta, Ecuador.

\section{Editor de Sección}

Dra. Fanny Miriam Sanabria-Boudri, Universidad Nacional Enrique Guzmán y Valle, Perú.

\section{Editor Técnico}

Lic. Henry Darío Suárez-Vélez, Polo de Capacitación, Investigación y Publicación (POCAIP), Manta, Ecuador.

\section{Editores Asociados}

Dra. Lenys Piña-Ferrer, Universidad Rafael Belloso Chacín, Maracaibo, Venezuela.

Dra. Ana Cristina-Chirinos, Universidad Nacional Experimental Francisco de Miranda, Venezuela, Bolivarian Republic of.

Mg. Danny E. Alcívar-Vélez, Universidad Particular San Gregorio de Portoviejo, Ecuador

\section{Editor Académico}

Dra. Juana Cecilia-Ojeda, Docente de la Universidad del Zulia, Maracaibo, Venezuela.

\section{Comité Científico}

Dra. Maritza Berenguer- Gouarnaluses, Universidad Santiago de Cuba, Santiago de Cuba, Cuba.

Dr. Félix Colina-Ysea, Universidad Privada SISE, Perú.

Dr. Lucio Noriero-Escalante, Universidad Autónoma de Chapingo, México. 
Dr. Oswaldo Zambrano-Quinde,

Universidad Laica Eloy Alfaro de Manabí, Ecuador, Manta, Ecuador.

$\mathrm{PhD}$. Lenin A. Párraga-Zambrano, Universidad Laica Eloy Alfaro de Manabí, Extensión Chone, Chone, Ecuador.

$\mathrm{PhD}$ José María Lalama-Aguirre, Universidad Central del Ecuador, Quito, Ecuador.

Dr. Gustavo J. Machado-Macellaro, Universidad de la República, Uruguay.

Dra. Elsy Vianelly González de Hernánde, Universidad Nacional Experimental del Yaracuy, Venezuela.

Dra. Mayra Elena Medina Medina, Universidad Pedagógica Experimental Libertador. Instituto Pedagógico Rural "Gervasio Rubio". IPRGR, Venezuela.

$\mathrm{Ph}$. D. Fernando Represa-Pérez, Universidad Laica "Eloy Alfaro" de Manabí, Manta, Ecuador.

Ph.D. Marlery Sánchez-Díaz, Universidad de la Habana, Cuba.

Dr. Jaime E. Cedeño-Zambrano, Casa Editora del Polo (CASEDELPO), Manta, Ecuador, Ecuador.

MSc. Darwin Alex Roldan-Mendoza, Casa Editora del Polo (CASEDELPO), Manta, Ecuador, Ecuador.

MSc. Marcos Boanerge Vera-Mendoza, Universidad Laica "Eloy Alfaro" de Manabí, Manta, Ecuador.
MSc. Pedro Enrique Reyes-Vélez, Universidad Laica "Eloy Alfaro" de Manabí, Manta, Ecuador.

\section{EQUIPO TÉCNICO}

\section{Diagramador}

Lic. Jessica María Mero-Vélez, Centro de Capacitación, Investigación y Publicación, CEICAPU, Manta, Ecuador.

\section{Diseñador}

Ing. Edwin A. Delgado-VélIz, Polo de Capacitación, Investigación y Publicación (POCAIP), Ecuador.

\section{Desarrollador Web}

Lic. Rodolfo Antonio Camacho-Albarrán, Instituto de Investigación y Estudios Avanzados Koinonía. Fundación Koinonía, Venezuela, Bolivarian Republic of.

Alejandro David Plúa-Argoti, Consultor, Casa Editora del Polo (CASEDELPO), Manta, Ecuador, Ecuador.

\section{Asistentes}

Tec. Juan Arturo Berenguer-Gouarnaluses, Universidad de Ciencias Médicas, Santiago de Cuba, Cuba, Cuba

\section{Filóloga}

Lic. Nora Núñez-Gollot, Casa Editora del Polo, Manta, Ecuador.

Traductor

Maydali Engracia Del Carmen VillasmilYánez, Universidad Nacional Experimental Francisco de Miranda, Venezuela, Ecuador. 